\title{
Preliminary study for fully digitally guided implant treatment with narrow implants in the anterior region
}

\author{
Jae-Seok Kang ${ }^{1}$, Hye-yun $\mathrm{Heo}^{2}$, and Mee-Kyung Son ${ }^{3 *}$ \\ ${ }^{1}$ Doctor of Dental Clinic, Department of Implant-Prosthodontics, Yedam Dental Hospital, Mokpo, Republic of Korea \\ ${ }^{2}$ Resident, Department of Prosthodontics, Collage of Dentistry, Chosun University, Gwangju, Republic of Korea \\ ${ }^{3}$ Professor, Department of Prosthodontics, Collage of Dentistry, Chosun University, Gwangju, Republic of Korea
}

\begin{abstract}
The aim of this study was to evaluate the survival rates of narrow implants placed in the maxillary or mandibular anterior region with digitally guided flapless surgery and prosthetic system. Thirty-five narrow implants were placed in the narrow anterior region in 20 patients using the digitally guided flapless surgery. The study subjects were divided into the immediate-loading group and the delayed-loading group. In 3 months, when the alveolar bone was healed, an oral scanner was used to produce zirconia prosthesis, and the final prosthesis was designed with a three-dimensional program. The degree of bone resorption around the implant was recorded at 12 months and 24 months after surgery. The implant fixation stability was measured after placement; the stability was in the range of Implant stability quotient (ISQ) 63-78 (average ISQ, 71.20 \pm 3.80 ). The radiographs obtained at 12 months and 24 months after implantation revealed that the bone around the implant in the fresh extraction socket and around the implant placed in the healed site showed an average bone resorption of $0.33 \pm 0.07 \mathrm{~mm}$ and $0.18 \pm 0.02 \mathrm{~mm}$, respectively. After 2 weeks, one implant was removed because the patient complained of pain with radiolucency in the entire fixture. The overall implant survival rate was $97.1 \%$. Narrow implants placed on the fresh extraction socket or healed site using the digitally guided surgery system were maintained well, with minor marginal bone resorption. As a result, we found that this method is useful for treating anterior teeth on a narrow bone.
\end{abstract}

Key Words: Digitally guided, Flapless surgery, Narrow diameter implant, Survival rate

(c) This is an open-access article distributed under the terms of the Creative Commons Attribution Non-Commercial License (http://creativecommons.org/licenses/by-nc/4.0) which permits unrestricted noncommercial use, distribution, and reproduction in any medium, provided the original work is properly cited.

\section{Introduction}

Restoring an area where a tooth has been lost using an implant is common prosthetic treatment. However, the narrow bone width of the maxillary lateral incisors or mandibular incisors makes it difficult to replace them with implants of standard diameter. In such cases, the use of a mallet or accompanying bone graft is recommended to in- crease the survival rate [1].

In general, numerous studies have reported the use of implants with a diameter of 3.0-3.5 $\mathrm{mm}$, which are "narrow implants'. Narrow implants are useful for the maxillary anterior or mandibular anterior region with a narrow buccolingual bone and the mandibular anterior region with insufficient space between teeth and they have also been successfully applied to the premolar or molar region [2].

Received August 17, 2020; Revised September 11, 2020 Accepted September 11, 2020

*Corresponding author: Mee-Kyoung Son, Department of Prosthodontics, College of Dentistry, Chosun University, 303 Pilmun-daero, Donggu, Gwangju 61452, Republic of Korea.

Tel: +82-62-220-3820, Fax: +82-62-227-7811, E-mail: son0513@chosun.ac.kr 
Implants with a diameter of less than $3.0 \mathrm{~mm}$ are generally manufactured as a single piece owing to problems in the manufacturing process.

When using them in the maxillary anterior region, abutments part should be prepared. If the implant placement direction is different from the prosthesis manufacturing direction, it can make implant prosthesis retention problem. In addition, abutments with a small diameter make it difficult to produce an aesthetic prosthesis and the phenomenon of the implant fixture bending under biting force has been reported by experiments [3].

Currently, with the advancement in processing technology and implant surface, two-piece narrow implants with the fixture and abutment manufactured separately, have been developed and applied clinically for implants of 3.0$3.3 \mathrm{~mm}$ in diameter. Two-piece implants have some advantages: an angled stock abutment or a custom abutment can be used in case of different insertion path between fixture and prosthesis and a digital impression can be acquired using a scan body or an oral scanner.

Since the implant platform surrounding the abutment is thin for a two-piece narrow implant, the implant placement torque is recommended to be $35 \mathrm{Ncm}$ or lower and the abutment fastening torque is recommended to be 20 $\mathrm{Ncm}$ or lower in order to prevent tearing in the top part of the implant and screw fracture (manufacturer recommendation).

In 2000, the three-dimensional (3D)-guided surgery system was first introduced in the field of implant dentistry called as 'Navigation' [4]. Since then, various digitally guided surgery systems have been introduced with much research and effort.

Recently, the popularity of Cone-Beam Computed Tomography (CBCT) and oral scanners in dentistry has enabled patient information to be easily converted into 3D data for implant surgery. Using these data and the programs, it is possible to predict virtual implant surgery and final prosthesis in advance. The diagnosis and design program enables us to predetermine the location, diameter, and length of the implant to be placed. If the initial fixation is stable after placement, it can be loaded immediately using pre-made custom abutment and temporary teeth. This not only reduces the amount of time taken to perform the surgery but also provides the patient with psychological satisfaction by solving the esthetic-related problems in the anterior region due to tooth loss.

In general, the little bone resorption was occurred when open the flap to place implant. In some case, thin bone(less than $1 \mathrm{~mm}$ ) was remained after placement of fixture in narrow (bucco-lingual) anterior area under the flap surgery. Unless accompanied by bone graft, the implant surface may be exposed cause of this resorption during osseointegration, resulting in failure. You et al. [5] reported relatively low bone resorption when the implant was placed without opening the flap using animal models.

The digitally guided surgery system enables the implant to be placed safely in a planned position without opening the flap. Recently, with the introduction of a high-precision digitally guided surgery system, it is possible to place a narrow implant in an accurate position without opening the flap in such regions with a narrow bone [6].

With the spread of oral scanners and the development of digital programs in dentistry, we can take a digital impression with this oral scanner and scan body for temporary or final prosthesis instead of analog coping impression without stone model. Also, with the help of the scan body, the final prosthesis can be designed by selecting the stock abutment or a custom abutment scanning to fabricate the final prosthesis.

This study aimed to report the results obtained with narrow implants for maxillary lateral incisors and mandibular anterior teeth using a digitally guided flapless surgery system.

\section{Materials and Methods}

This clinical study was performed retrospectively and reviewed 20 patients who visited Yedam dental hospital from 2016 to 2019 for placement of narrow implant (UFII; DIO Implant, Busan, Korea) with diameters of $3.0 \mathrm{~mm}$ and 3.3 $\mathrm{mm}$ in the maxillary anterior or mandibular anterior region. Patients with a history of systemic diseases and conditions including diabetes, cardiovascular diseases, autoimmune diseases, malignancies, blood-related diseases, osteoporosis, smoking, parafunctional habits (clenching and bruxism) were excluded from the study. Total 35 fixtures were 
placed. Patient's data including the gender, age, placement site, implant diameter, implant length, placement in a fresh extraction socket or healed site, initial fixation stability (Implant stability quotient, ISQ), and immediate loading or delayed loading were categorized and recorded in files (Microsoft Excel 2007; Microsoft, Redmond, WA, USA) for evaluation. Radiographic examination for evaluation was performed for 2 years after implant placement at 6-month intervals, with reference to the data before surgery. Alveolar bone loss was measured using baseline and follow-up periapical radiographs. To assess bone level in the periapical radiographs, distance between distal and mesial bone margins and implant shoulder was measured by digital caliper and their mean value was recorded. Each patient underwent a full-body medical check-up to check for any etio-

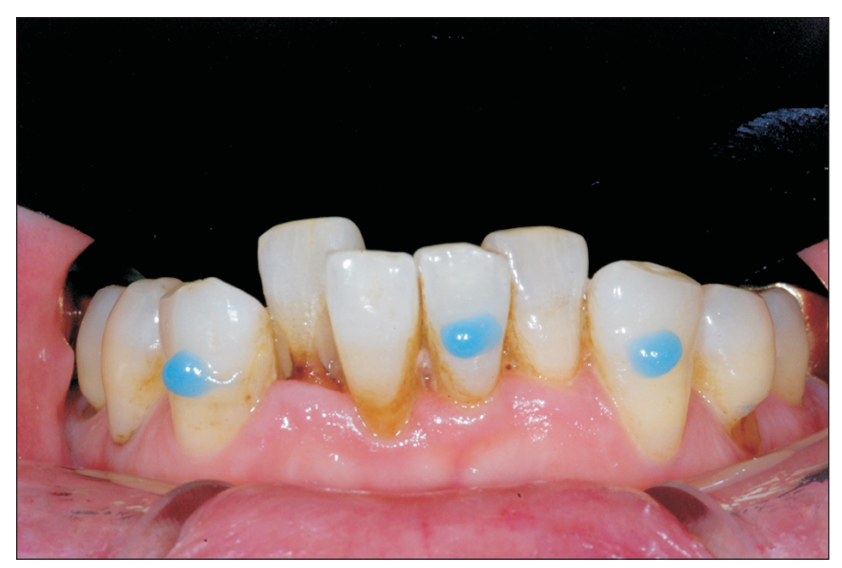

Fig. 1. Blue resin attached to the mandibular anterior teeth prior to oral scanning for accurate merging. logical factors that could affect implant surgery outcomes.

\section{Guide design with Implant Studio (3 Shape) and custom design with Dental System (3 Shape)}

For implant placement planning and surgical guide fabrication, the oral data of all patients were obtained using a Trios 3 (3 Shape, Copenhagen, Denmark) intraoral scanner, and CBCT (PHT-30LFO; Vatech, Hwaseong, Korea) examination was carried out for alveolar bone data. For accurate merging of the oral data and CBCT data, blue color composite resin (Temp Flow; DENTEX, Shenzhen, China) was attached to adjacent teeth as a merging point before obtaining the oral scanner data and CBCT data (Fig. 1, 2). After merging the data using Implant Studio (3 Shape) and determining the adequate implant position, diameter, and length for the surgical site, a surgical guide was designed.

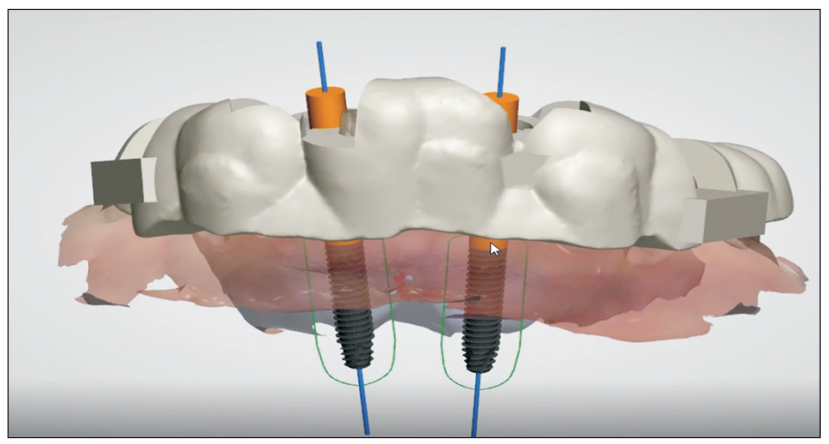

Fig. 3. Surgical guide design for a narrow implant in Implant Studio (3 Shape).

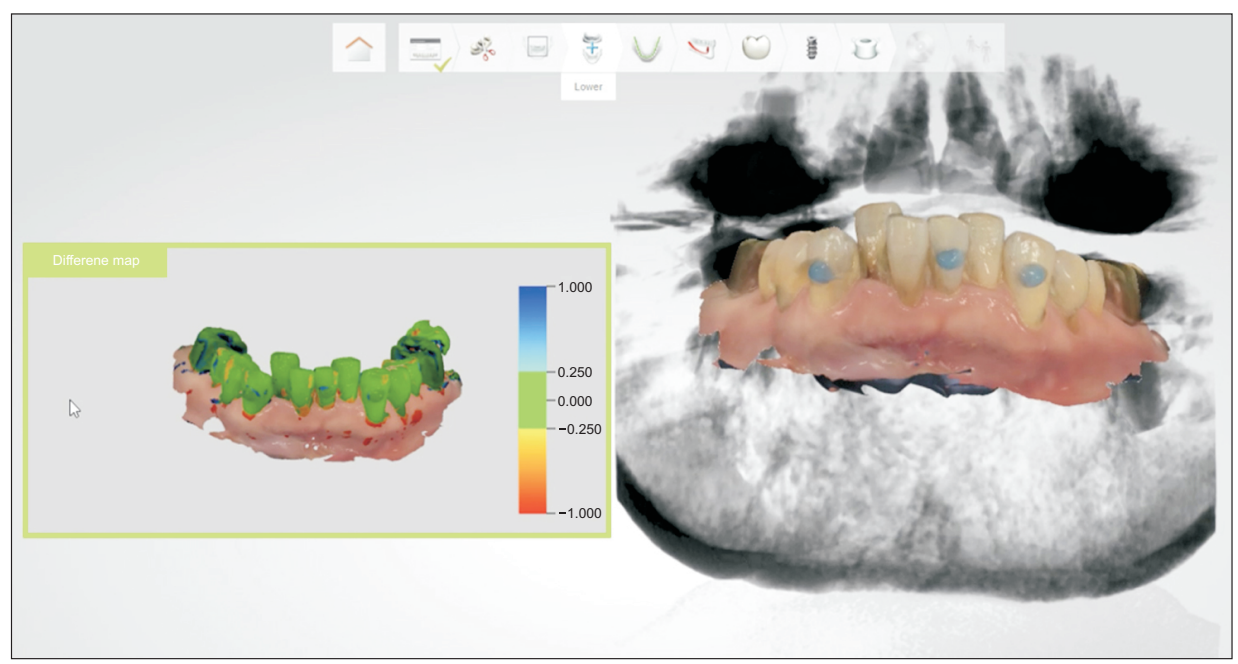

Fig. 2. Oral scanning and CBCT data accurately merged in Implant Studio (3 Shape). 
Using these data, the custom abutment and temporary tooth for immediate loading were designed using Dental System (3 Shape) (Fig. 3). The guide, temporary tooth, and jig designed for surgery were resin printed using an MJP 3D printer (3D Systems; Rock Hill, SC, USA) or a DLP 3D printer (Probo; DIO implant), and the custom abutment was prepared by commissioning CNC lathe processing (Fig. 4).

\section{Preparation of surgery site and atraumatic extraction}

As long as there was no unusual medical history, on the day of surgery, $0.12 \%$ chlorohexidine was used to clean the mouth for 1 minute for intraoral disinfection, and infiltrating anesthesia using 4\% lidocaine (1:100,000 adrenaline) was performed in the maxillary palate, the alveolar crest, and the maxillary and mandibular vestibule regions. If the surgical site required extraction for immediate placement, a non-traumatic and safe extraction procedure was essential for preserving the alveolar bone and gingiva, using a surgical bur, a pair of forceps for the anterior teeth, and a luxating elevator (root picker). When separating the residual roots with surgical bur, it is recommended to cut them bucco-lingual direction and separate it mesio-distally to protect the alveolar bone for safe initial fixation upon immediate placement and to protect the aesthetically important buccal bone.

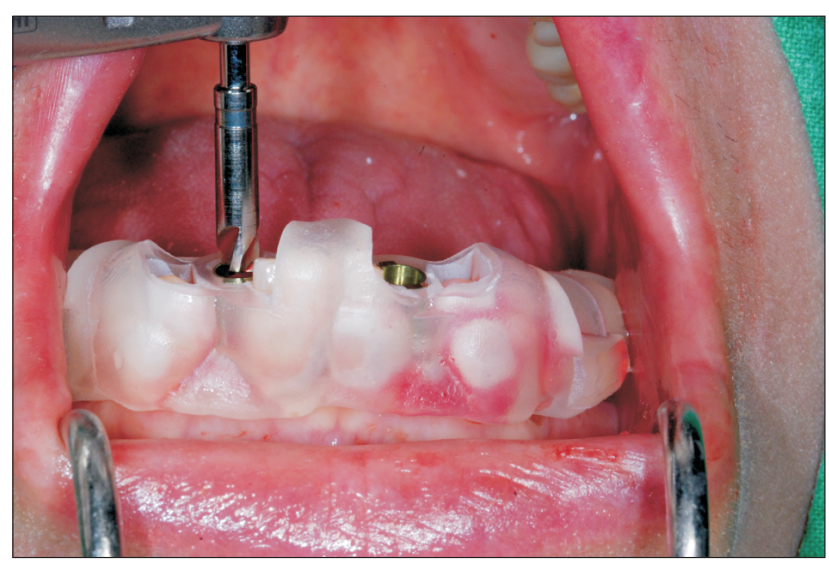

Fig. 4. Long sleeve and long drill system for narrow bone.

\section{Drilling and installation of fixture}

After confirming the stability of the surgical guide in the fresh extraction socket or healed site, Flapless implant placement surgery was done using a dedicated kit for narrow implants. Pre-determined narrow fixtures (UFII, DIO Implant) of $3.0-3.3 \mathrm{~mm}$ in diameter and $11.5-13 \mathrm{~mm}$ in length were used (Fig. 5). All drilling procedures were performed according to a pre-determined sequence, and the drilling was proceeded at a speed of less than $100 \mathrm{rpm}$ to suppress heat generation. Between each drilling, the drilling site was cleaned and cooled by sufficient irrigation. In order to accurately connect the pre-made abutment, it must be placed at a pre-planned height (offset), and it need of horizontal hex alignment between fixture and abutment. An implant placement torque of $40 \mathrm{Ncm}$ or lower is recommended to prevent deformation of the weak internal structure.

\section{Immediate loading with custom abutment and temporary crown}

The ISQ value with Osstell Mentor was measured for immediate loading on the placed fixture, and an early load was applied only on the fixture with an ISQ value of 70 or higher. For immediate loading, custom abutment was connected to fixture by jig or temporary teeth for preventing rotation of the pre-positioned fixture (Fig. 6). The tightening torque was also maintained at $20 \mathrm{Ncm}$ or lower to

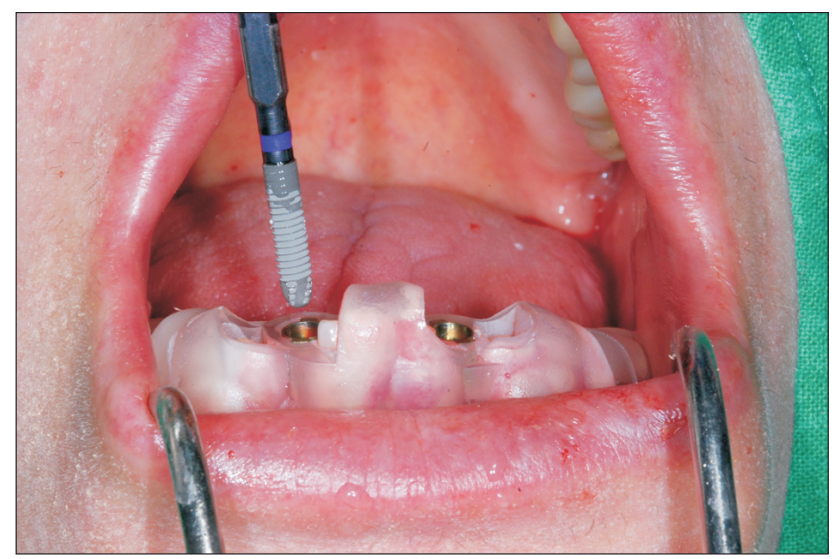

Fig. 5. Diameter 3.3 narrow implant fixtures (UFII, DIO implant, Busan, Korea). 


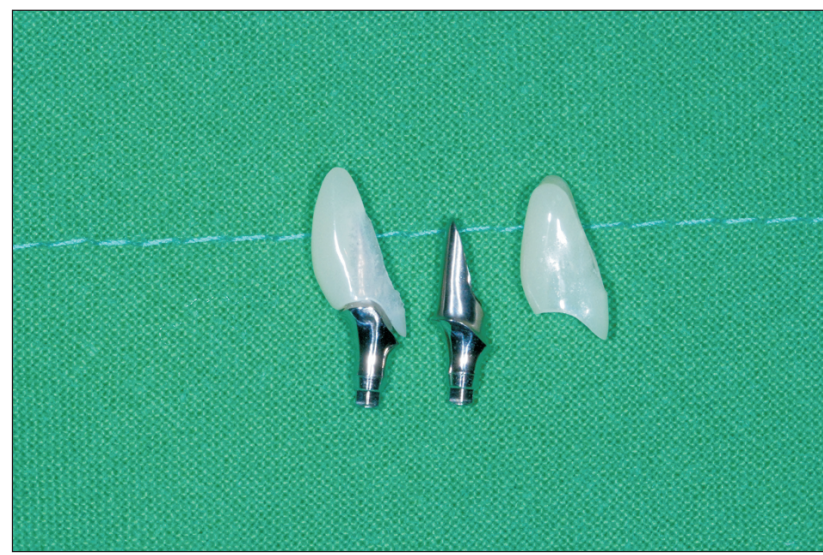

Fig. 6. Custom abutment and temporary crown prepared for early loading.

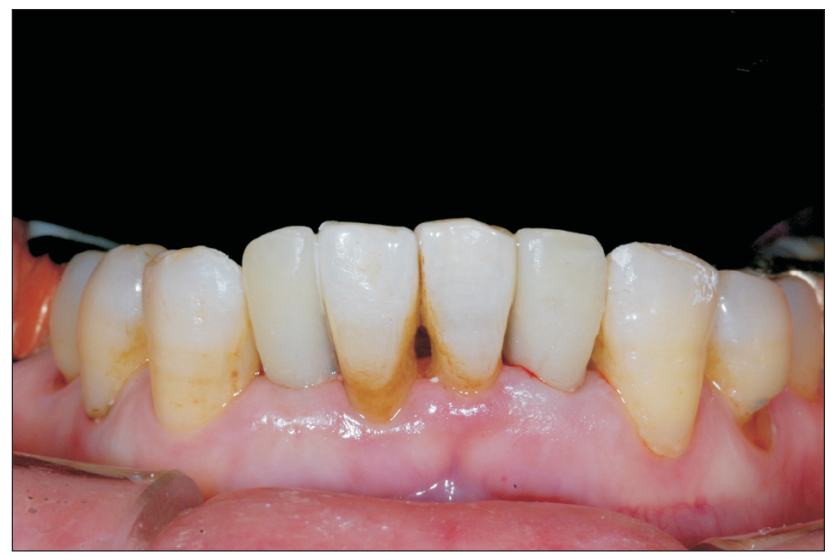

Fig. 7. Early loading using temporary crown.

prevent fracture of the connecting screw. When sufficient initial stability was not achieved, a healing abutment was connected to prevent the loading while osseointegration (Fig. 7). After placing all implants, panoramic and standardized periodical radiographs and $\mathrm{CBCT}$ images were taken.

\section{Intraoral scanning of modified custom abutment for final prosthesis}

Two-to-three months after implant placement, when osseointegration was completed, the final prosthesis was designed and fabricated using an Trios 3 intraoral scanner and Dental System, and all prostheses were prepared using the 3D digital information without modeling. If the load was not immediately applied, a custom abutment and a final prosthesis were produced using a scan body. We took a

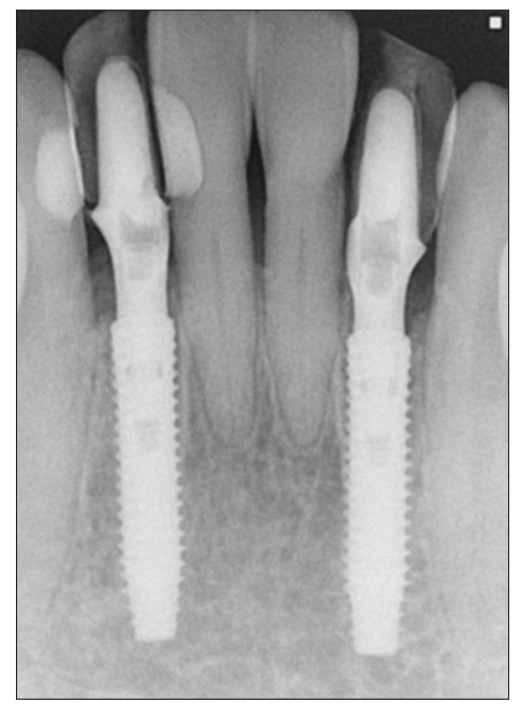

Fig. 8. Standardized periapical radiograph taken after early loading.

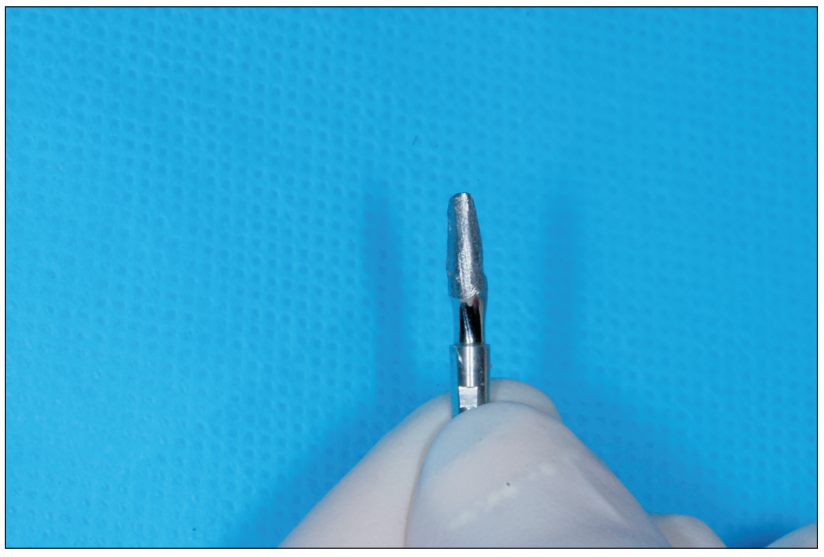

Fig. 9. Scanning with Trios 3 (3 Shape) after connecting to analog for marginal adjustment of the custom abutment from the outside of the oral cavity.

standardized periapical radiograph after early loading (Fig. 8). If the used custom abutment margin had been exposed because of soft tissue retraction while osseointegration since immediate loading, the margin was adjusted from the inside and outside of the oral cavity by re-scanning with intra oral scanner (Fig. 9).

The STL files obtained by the intraoral scanner were transferred to Dental System, and the custom abutment images taken from outside of the oral cavity were used as a library to be matched with each intraoral custom abutment images for production of the final prosthesis (Fig. 10).

At this time, dental technician could see exact abutment 


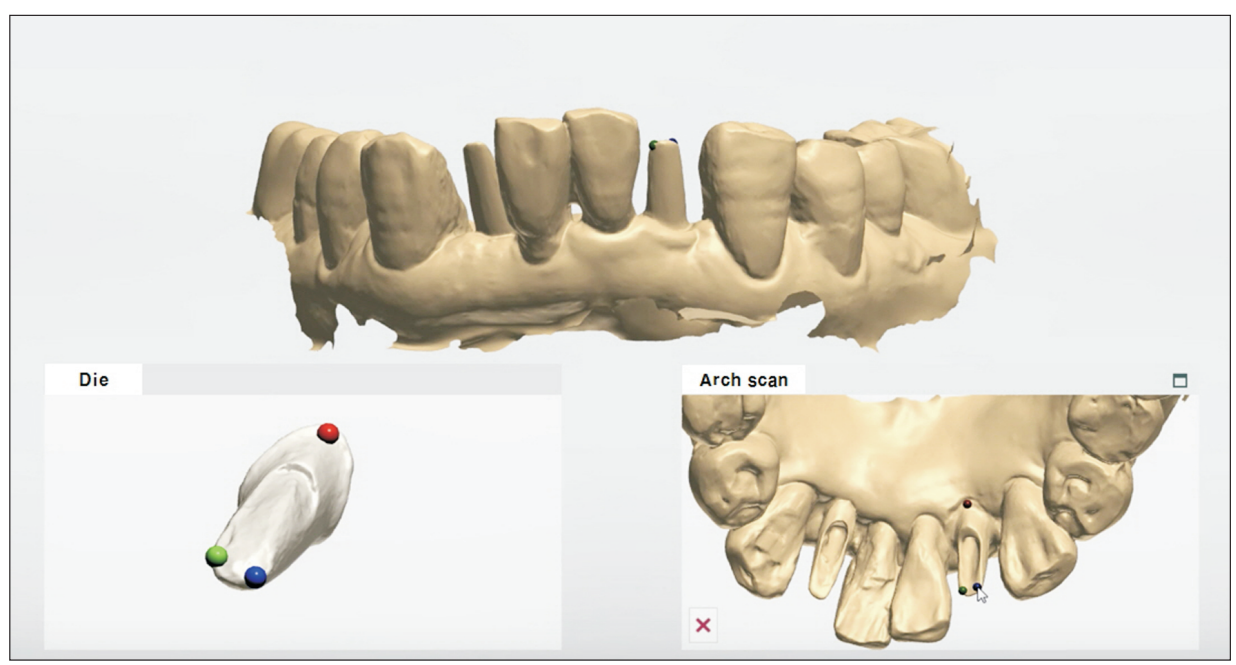

Fig. 10. Matching the custom abutment file from the outside of the oral cavity with the intraoral scanned custom abutment in Dental system (3 Shape).

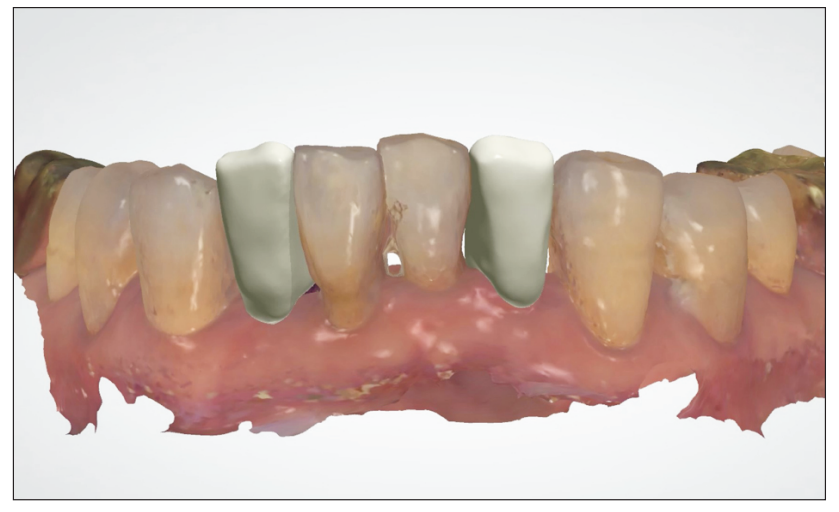

Fig. 11. Design of the final prosthesis without model.

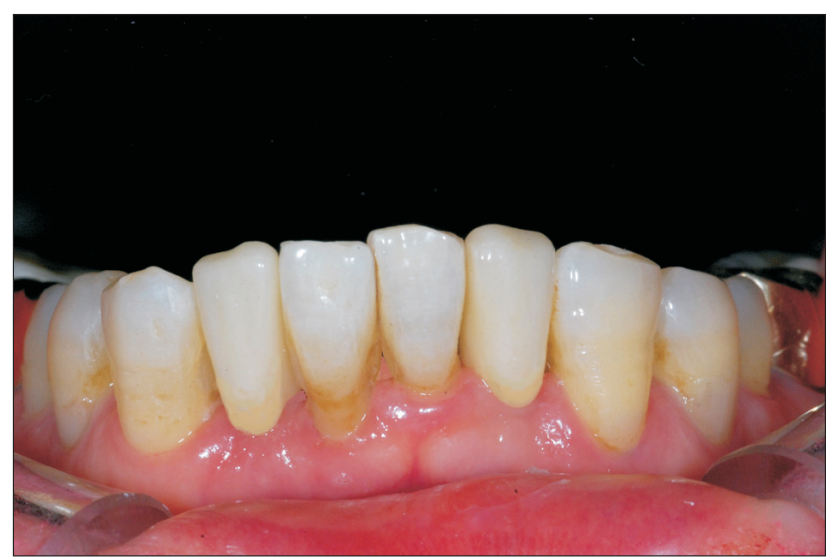

Fig. 12. Cemented zirconia final prosthesis in the oral cavity.

margin on Dental System program for final prosthesis. The final prosthesis was made of monolithic zirconia and sent to the clinic after sintering and coloring. The final prosthe-

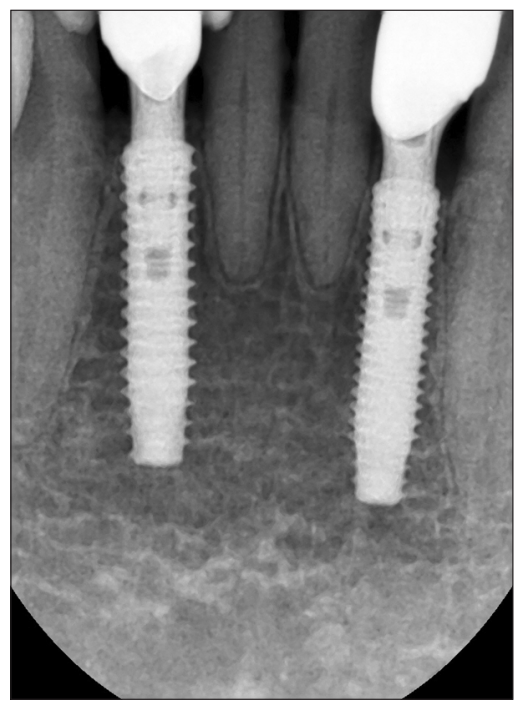

Fig. 13. Standardized periapical radiograph taken 24 months after immediate loading.

sis was accurately fit over the custom abutment even without a model, and after temporary adhering, it was finally cemented after one month of adaptation (Fig. 11,12). Afterwards, the patients were followed up every 6 months, and radiographs were taken after 12 and 24 months to evaluate marginal bone resorption (Fig. 13).

\section{Results}

The 20 patients, 11 males and 9 females, in this study had no medical history that could affect the surgery, and the age of the patients ranged from 28 to 67 years. Of the 35 
narrow implants placed, 7 were placed in the maxillary anterior region, and 28 were placed in the mandibular anterior region. In total, twenty 3.0-mm diameter implants and fifteen 3.3-mm diameter implants were placed. Fifteen implants out of thirty-five were $11.5 \mathrm{~mm}$ in length, and other 20 implants were $13 \mathrm{~mm}$ in length. Thirteen implants were placed in fresh extraction sockets, accounting for $37.1 \%$ of the total, and 22 implants were placed in healed sites, accounting for $62.8 \%$.

Twenty-three implants (65.7\%) were connected with the abutment right after placement for immediate loading, and the prostheses were produced upon osseointegration. The ISQ of the implant measured after placement was in the range of ISQ 63 to 78 , and the average value was ISQ 71.20 \pm 3.80 . The average ISQ of the implant eligible for immediate loading was ISQ $73.17 \pm 2.27$ (Table 1).

To measure marginal bone resorption, the standardized periodical radiographs taken after implant placement and the radiographs taken after 12 and 24 months were converted into high-resolution JPEG files, and the first bone-to-implant contact distance from the shoulder of the implant was measured using view rulers in Photoshop to observe the changes.

The bone around the implant placed in the fresh extraction sockets showed an average bone resorption of $0.33 \pm$ $0.07 \mathrm{~mm}$, and the bone around the implant placed in the normal healed sites showed an average bone resorption of $0.18 \pm 0.02 \mathrm{~mm}$ (Table 1). Two weeks after implant placement, one implant out of all implants placed was removed as the patient complained of pain with radiolucency in the entire fixture. The overall implant survival rate was $97.1 \%$.

\section{Discussion}

Many studies have shown that narrow or small-diameter implants have a lower survival rate than regular-diameter implants and that implant diameter acts as an important factor for successful outcomes, with $3.0 \mathrm{~mm}$ as the standard diameter. Therefore, implants with a diameter of $3.0 \mathrm{~mm}$ or smaller have been reported to be associate with the risk of failure $[7,8]$. However, relatively high survival rates have been reported for implants with a diameter of $3.0 \mathrm{~mm}$ or larger. In 2008, Zinsli et al. [9] reported a high survival rate using 3.3-mm diameter implants. In particular, it has been reported that a narrow diameter implant does not cause pathological problems even if it is placed within $1 \mathrm{~mm}$ of the adjacent teeth [10].

In this study, 35 narrow implants of $3.0 \mathrm{~mm}$ and $3.3 \mathrm{~mm}$ in diameter were placed in the maxillary anterior or mandibular anterior region and observed for 2 to 3 years, showing a survival rate of $97.1 \%$ as a result. A failed implant was removed within 2 weeks from the placement due to pain and fast bone resorption observed in the radiograph.

Peron and Romanos [11] reported changes within 0.5 $\mathrm{mm}$ over 2 years after placing 16 narrow implants in extraction and healed sites for immediate loading. This study also observed bone resorption under $0.5 \mathrm{~mm}$ upon keeping track of the changes in the bone around the implants for 2 to 3 years, with resorption of $0.33 \pm 0.07 \mathrm{~mm}$ for the implants placed in fresh extraction sockets for immediate loading and resorption of $0.18 \pm 0.02 \mathrm{~mm}$ for the implants placed in healed sites. One of the reason of these difference is thought to be due to the bone graft within the jumping distance [12] in the gap between the implant platform and the bone around the heeling socket. In this study, there was a difference in bone resorption rate between immediate and delayed loads, but statistical analysis was not performed because the number of individuals in each group was small and the number of individuals between groups was not the same. Therefore, there was a limitation in evaluating the difference between the two groups except that absorption of less than $0.5 \mathrm{~mm}$ occurred in both groups.

Table 1. The comparison of average marginal bone loss and ISQ

\begin{tabular}{lccccc}
\hline \multirow{2}{*}{ Value } & \multicolumn{2}{c}{ Marginal bone loss } & & \multicolumn{2}{c}{ ISQ } \\
\cline { 2 - 3 } \cline { 5 - 6 } & Immediate placement $(\mathbf{m m})$ & Late placement $(\mathbf{m m})$ & & Immediate loading & Delayed loading \\
\hline \multirow{2}{*}{ Average } & 0.33 & 0.18 & 73.17 & 67.67 \\
SD & 0.07 & 0.02 & 2.27 & 3.87 \\
\hline
\end{tabular}

ISQ, Implant stability quotient; SD, standard deviation. 
Han et al. [13] recommended immediate loading with an insertion torque of $40 \mathrm{Ncm}$ or more and ISQ 70 or higher upon performing immediate loading in partially edentulous mandible in their systemic review. The average ISQ of implants placed with a $40 \mathrm{Ncm}$ insertion torque using a digitally guided system was $71.20 \pm 3.80$ in this study. The average ISQ of the immediate loading group was $73.17 \pm$ 2.27 .

The main problem with the introduction of the digitally guided surgery system was the deviation before and after the surgery, and various results using various systems have been published. In light of the reported statistics, the average linear deviation was $1.22 \mathrm{~mm}$ in the platform and 1.51 $\mathrm{mm}$ in the apex area, and the average angular deviation was 4.9 degrees [14-17].

Many attempts have been made to improve the precision of the digitally guided surgery system. Kholy et al. [18] reported that precision could be improved by reducing the drilling distance to the sleeve by adjusting the length of the sleeve or by reducing the length of the implant. Fang et al. [6] reported the average linear deviation of $0.46 \mathrm{~mm}$ in the implant platform, $0.67 \mathrm{~mm}$ in the apex area, and the angular deviation of 1.40 degrees, on comparing 40 implants placed in the anterior region of 32 patients using a digitally guided surgery system (DIO implant) for narrow implants, equipped with a long tube developed for accurate long drilling and precise placement of narrow implants.

When using the digitally guided surgery system, heat is generated during the drilling process, which may cause bone necrosis after surgery. In this study, drilling was performed at 100 RPM or less with sufficient irrigation to prevent heat generation.

The guided surgery system used in this study also used a long drill and a long tube to increase precision, and a custom abutment and PMMA temporary crown were used for immediate loading.

For producing the final prosthesis, the custom abutment was removed from the fixture to adjust the margin and shape after removing the temporary teeth that achieved osseointegration, scanned using the intraoral scanner to save as data and remounted on the fixture to scan the entire set of teeth and occlusion. The STL files obtained by the intra oral scanner were transferred to Dental System (3 Shape), and the custom abutment images taken from outside the oral cavity were used as a library to be matched with each intra oral custom abutment images to design the final prosthesis.

It was possible to design the final prosthesis in the program could clearly distinguish the marginal area located below the gingiva. The final prosthesis was fabricated using a zirconia block and completed with coloring. All of the processes were produced as digital processes without model production.

In previous studies, several authors reported that the use of narrow-diameter implants increased the risk of prosthetic complications or implant fracture $[9,19]$ but no such phenomenon was observed in this study. Further studies with more cases and longer follow-up will be needed to confirm the results of this clinical study.

\section{Acknowledgements}

This study was supported by a research fund from Chosun University, 2019. The authors wish to thanks to Dental technician Il Young Park in Yedam CAD/CAM center.

\section{Conflicts of Interest}

The authors declare that they have no competing interests.

\section{ORCID}

\author{
Jae-Seok Kang \\ https://orcid.org/0000-0003-0419-2559 \\ Hye-Yun Heo \\ https://orcid.org/0000-0003-2378-1367 \\ Mee-Kyoung Son \\ https://orcid.org/0000-0001-9225-1744
}

\section{References}

1. Yang G, Chen L, Gao Y, Liu H, Dong H, Mou Y. Risk factors and reoperative survival rate of failed narrow-diameter implants in the maxillary anterior region. Clin Implant Dent Relat Res 2020;22:29-41. doi: 10.1111/cid.12867.

2. Alrabiah M, Al Deeb M, Alsahhaf A, AlFawaz YF, Al-Aali KA, Vohra F, Abduljabbar T. Clinical and radiographic 
assessment of narrow-diameter and regular-diameter implants in the anterior and posterior jaw: 2 to 6 years of follow-up. J Periodontal Implant Sci 2020;50:97-105. doi: 10.5051/jpis.2020.50.2.97.

3. Neto TH, Tuzia AS, Gehrke SA, Moura RDV, Casati MZ, Mesquita AMM. A comparative analysis of implants presenting different diameters: extra-narrow, narrow and conventional. Materials (Basel) 2020;13:1888. doi: 10.3390/ma13081888.

4. Jung RE, Schneider D, Ganeles J, Wismeijer D, Zwahlen M, Hämmerle $\mathrm{CH}$, Tahmaseb A. Computer technology applications in surgical implant dentistry: a systematic review. Int J Oral Maxillofac Implants 2009;24 Suppl:92-109. doi: 10.5167/uzh-26119.

5. You TM, Choi BH, Li J, Xuan F, Jeong SM, Jang SO. Morphogenesis of the peri-implant mucosa: a comparison between flap and flapless procedures in the canine mandible. Oral Surg Oral Med Oral Pathol Oral Radiol Endod 2009;107:66-70. doi: 10.1016/j.tripleo.2008.05.045.

6. Fang Y, An X, Jeong SM, Choi BH. Accuracy of computerguided implant placement in anterior regions. J Prosthet Dent 2019;121:836-842. doi: 10.1016/j.prosdent.2018.07.015.

7. Allum SR, Tomlinson RA, Joshi R. The impact of loads on standard diameter, small diameter and mini implants: a comparative laboratory study. Clin Oral Implants Res 2008;19:553-559. doi: 10.1111/j.1600-0501.2007.01395.x.

8. Schiegnitz E, Al-Nawas B. Narrow-diameter implants: a systematic review and meta-analysis. Clin Oral Implants Res 2018;29:21-40. doi: 10.1111/clr.13272.

9. Zinsli B, Sägesser T, Mericske E, Mericske-Stern R. Clinical evaluation of small-diameter ITI implants: a prospective study. Int J Oral Maxillofac Implants 2004;19:92-99.

10. Trbakovic A, Bongenhielm U, Thor A. A clinical and radiological long-term follow-up study of narrow diameter implants in the aesthetic area. Clin Implant Dent Relat Res 2018;20:598-605. doi: 10.1111/cid.12627.

11. Peron C, Romanos G. Immediate provisionalization of single narrow implants in fresh extraction sockets and healed sites: clinical and radiographic outcomes of 2 years follow- up. Int J Periodontics Restorative Dent 2020;40:417-424. doi: 10.11607/prd.4622.

12. Botticelli D, Berglundh T, Buser D, Lindhe J. The jumping distance revisited: an experimental study in the dog. Clin Oral Implants Res 2003;14:35-42. doi: 10.1034/j.16000501.2003.140105.x.

13. Han CH, Mangano F, Mortellaro C, Park KB. Immediate loading of tapered implants placed in postextraction sockets and healed sites. J Craniofac Surg 2016;27:1220-1227. doi: 10.1097/SCS.0000000000002756.

14. Zhou W, Liu Z, Song L, Kuo CL, Shafer DM. Clinical factors affecting the accuracy of guided implant surgery-a systematic review and meta-analysis. J Evid Based Dent Pract 2018;18:28-40. doi: 10.1016/j.jebdp.2017.07.007.

15. Bover-Ramos F, Viña-Almunia J, Cervera-Ballester J, Peñarrocha-Diago M, García-Mira B. Accuracy of implant placement with computer-guided surgery: a systematic review and meta-analysis comparing cadaver, clinical, and in vitro studies. Int J Oral Maxillofac Implants 2018;33:101115. doi: 10.11607/jomi.5556.

16. Tahmaseb A, Wismeijer D, Coucke W, Derksen W. Computer technology applications in surgical implant dentistry: a systematic review. Int J Oral Maxillofac Implants 2014;29 Suppl:25-42. doi: 10.11607/jomi.2014suppl.g1.2.

17. Lin CC, Wu CZ, Huang MS, Huang CF, Cheng HC, Wang DP. Fully digital workflow for planning static guided implant surgery: a prospective accuracy study. J Clin Med 2020;9:980. doi: 10.3390/jcm9040980.

18. El Kholy K, Janner SFM, Schimmel M, Buser D. The influence of guided sleeve height, drilling distance, and drilling key length on the accuracy of static Computer-Assisted Implant Surgery. Clin Implant Dent Relat Res 2019;21:101107. doi: 10.1111/cid.12705.

19. Romeo E, Lops D, Amorfini L, Chiapasco M, Ghisolfi M, Vogel G. Clinical and radiographic evaluation of smalldiameter (3.3- $\mathrm{mm}$ ) implants followed for 1-7 years: a longitudinal study. Clin Oral Implants Res 2006;17:139-148. doi: 10.1111/j.1600-0501.2005.01191.x. 